\title{
On the geometry of the rotating liquid drop
}

\author{
Ivaiilo M. Mladenov John Oprea
}

\begin{abstract}
Here we consider the problem of a fluid body rotating with a constant angular velocity and subjected to surface tension. Determining the equilibrium configuration of this system turns out to be equivalent to the geometrical problem of determining the surface of revolution with a prescribed mean curvature. In the simply connected case, the equilibrium surface can be parameterized explicitly via elliptic integrals of the first and second kind. Here, we present two such parameterizations of the drops and we use the second of them to study finer details of the drop surfaces such as the existence of closed geodesics.
\end{abstract}

(C) 2014 IMACS. Published by Elsevier B.V. All rights reserved.

Keywords: Profile curves; Surfaces; Curvatures; Geodesics

\section{Introduction}

Let us consider a fluid body with incompressible mass rotating with a constant angular velocity and subjected to surface tension. The problem then is to find the shape which this mass will have under prescribed angular velocity.

The only force acting inside the drop is the centripetal force generated from the potential $\rho \omega^{2} r^{2} / 2$ and directed away from the axis. Here, $\rho$ is the fluid density, $\omega$ is the fixed angular velocity and $r$ is the radial distance from the axis of rotation. Because the fluid is assumed to be incompressible, a constant internal pressure $p_{i}$ results within the body. According to the Laplace-Young equation [9], at the free surface $\mathcal{S}$ enclosing the drop the surface tension $\sigma$ generates a pressure proportional to its mean curvature $H, \sigma H$. Finally, we must also add the constant pressure $p_{e}$ of the external fluid. The surface in equilibrium is therefore described by equating these pressures:

$$
p_{i}+\frac{\rho \omega^{2} r^{2}}{2}=\sigma H+p_{e}
$$

where $\omega, p_{e}, p_{i}, \rho, \sigma$ are constants. This then reduces immediately to the equation:

$$
H=2 \tilde{a} r^{2}+\tilde{c}
$$

where $\tilde{a}$ is a positive constant and $\tilde{c}$ is arbitrary. 


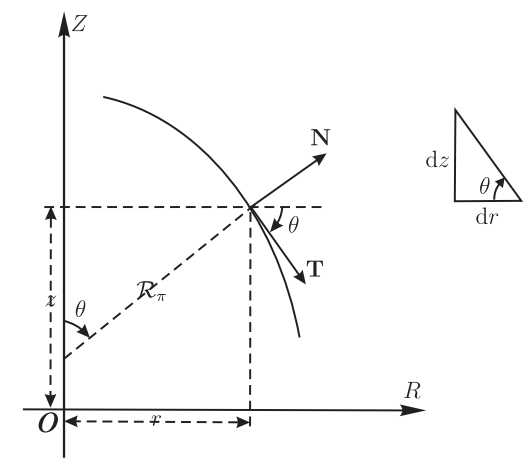

Fig. 1. Geometry of the profile curve.

\section{Geometry and surface invariants}

Let us now recall the well-known fundamental relations among meridional curvature $\kappa_{\mu}$, circumferential curvature $\kappa_{\pi}$ and mean curvature $H$ for surfaces of revolution [3],

$$
\kappa_{\mu}=\frac{\mathrm{d}\left(r \kappa_{\pi}\right)}{\mathrm{d} r}, \quad H=\frac{\kappa_{\mu}+\kappa_{\pi}}{2} .
$$

The simultaneous solution of the system of Eqs. (2) and (3) is

$$
\kappa_{\pi}=\tilde{a} r^{2}+\tilde{c}+\frac{C}{r^{2}}, \quad \kappa_{\mu}=3 \tilde{a} r^{2}+\tilde{c}-\frac{C}{r^{2}},
$$

where $C$ is an integration constant which will be assumed to be zero from now on. We make this assumption here in order to get analytical formulas for the rotating drop. At the moment, letting $C$ be non-zero only affords numerical analysis.

Moreover, if $\mathcal{R}_{\pi}$ is the distance from the surface point to the intersection of the normal at that point with the symmetry axes (see Fig. 1, where we use $\mathcal{R}$ for the abscissa simply to match the notation $\mathcal{R}_{\pi}$ ), then we have additionally,

$$
\kappa_{\pi}=\frac{1}{\mathcal{R}_{\pi}}=\frac{\sin \theta}{r},
$$

and therefore

$$
\frac{\mathrm{d} z}{\mathrm{~d} r}=-\tan \theta=-\frac{r \kappa_{\pi}}{\sqrt{1-\left(r \kappa_{\pi}\right)^{2}}} .
$$

Note that we take the convention that $\theta$ is measured from the horizontal line through the point and is positive when going clockwise. This means that $\theta$ will begin negative with the consequence that the derivative $\mathrm{d} z / \mathrm{d} r$ will be positive and the height of the curve will increase.

Finally, the ordinate of the profile curve is given by the integral

$$
z(r)=-\int \frac{\left(\tilde{a} r^{3}+\tilde{c} r\right) \mathrm{d} r}{\sqrt{1-\left(\tilde{a} r^{3}+\tilde{c} r\right)^{2}}} .
$$

Here, the indefinite integral produces an expression in $r$ that gives the $r$-dependent formula for $z$. From these considerations, we obtain a profile curve for the rotating drop, $(r, z(r))$ with

$$
\mathbf{x}(u, v)=(r \cos (v), r \sin (v), z(r)),
$$

being a parametrization for the surface of revolution $\mathcal{S}$ that is the drop itself. 
Having this result we can compute the coefficients of the first $(E, F, G)$ and second $(L, M, N)$ fundamental forms of $\mathcal{S}$ using Maple. ${ }^{1}$ The general formulas are as follows:

$$
\begin{aligned}
& E=\frac{1}{1-\left(\tilde{a} r^{3}+\tilde{c} r\right)^{2}}, \quad F=0, \quad G=r^{2}, \\
& L=\frac{3 \tilde{a} r^{2}+\tilde{c}}{1-\left(\tilde{a} r^{3}+\tilde{c} r\right)^{2}}, \quad M=0, \quad N=r^{2}\left(\tilde{a} r^{2}+\tilde{c}\right) .
\end{aligned}
$$

These quantities (also including Gaussian and mean curvatures) may be found by hand (with difficulty) or by using software such as Maple. Following the traditions of classical differential geometry [10], we use the parametrization of the drop given above with $u$ instead of $r$ :

$$
\mathbf{x}(u, v):=\left[\begin{array}{c}
u \cos (v) \\
u \sin (v) \\
\left(\tilde{a} u^{3}+\tilde{c} u\right) \mathrm{d} u \\
-\int \frac{\tilde{a}^{2} u^{6}-2 \tilde{a} \tilde{c} u^{4}-\tilde{c}^{2} u^{2}}{\sqrt{1-}}
\end{array}\right] .
$$

From the general expressions (8), we first find the metric coefficients to be

$$
[E, F, G]=\left[-\frac{1}{-1+\tilde{a}^{2} u^{6}+2 \tilde{a} \tilde{c} u^{4}+\tilde{c}^{2} u^{2}}, 0, u^{2}\right] .
$$

The coefficients of the second fundamental form are

$$
[L, M, N]=\left[-\frac{3 \tilde{a} u^{2}+\tilde{c}}{-1+\tilde{a}^{2} u^{6}+2 \tilde{a} \tilde{c} u^{4}+\tilde{c}^{2} u^{2}}, 0,\left(\tilde{a} u^{2}+\tilde{c}\right) u^{2}\right],
$$

and we immediately calculate the mean curvature,

$$
H=2 \tilde{a} u^{2}+\tilde{c},
$$

and the Gauss curvature

$$
K=\left(3 \tilde{a} u^{2}+\tilde{c}\right)\left(\tilde{a} u^{2}+\tilde{c}\right) .
$$

From the parametrization (or via a simple calculation with Gauss and mean curvatures), we also see that the principal curvatures are $\kappa_{\mu}=3 \kappa_{\pi}-2 \tilde{c}$, which makes the rotating drop a linear Weingarten surface. For more information about these, see $[4,7]$.

\section{Parameterization via Legendre's integrals}

When performing the integration in (7) it turns out to be convenient to switch from variables $\tilde{a}, \tilde{c}$ to variables $\tau, \stackrel{\circ}{r}$ defined by the relations:

$$
\tilde{a} \stackrel{\circ}{ }^{3}+\tilde{c} \stackrel{\circ}{r}=1, \quad \tau=\frac{1}{\tilde{a} r} .
$$

In view of (5) the first equation has an obvious geometrical interpretation as specifying the distance $\stackrel{\circ}{r}$ from the origin to the point on the equator where the tangent is normal to the abscissa. Now, by (4) and (5), we see that we have $\sin (\theta(\stackrel{\circ}{)}))=\stackrel{\circ}{r} \kappa_{\pi}=1$ and the geometrical relation (9) reduces to

$$
\theta\left(\stackrel{\circ}{)}=\frac{\pi}{2} .\right.
$$

\footnotetext{
${ }^{1}$ The Maple worksheet (which works with Maple 15) with the calculations below and with procedures for geodesics may be downloaded at academic.csuohio.edu/oprea_j/research/research.html.
} 


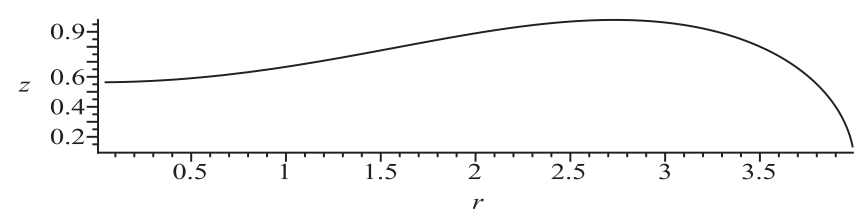

Fig. 2. A typical profile of the drop generated with $\stackrel{\circ}{r}=4$ and $\tau=0.533333$.

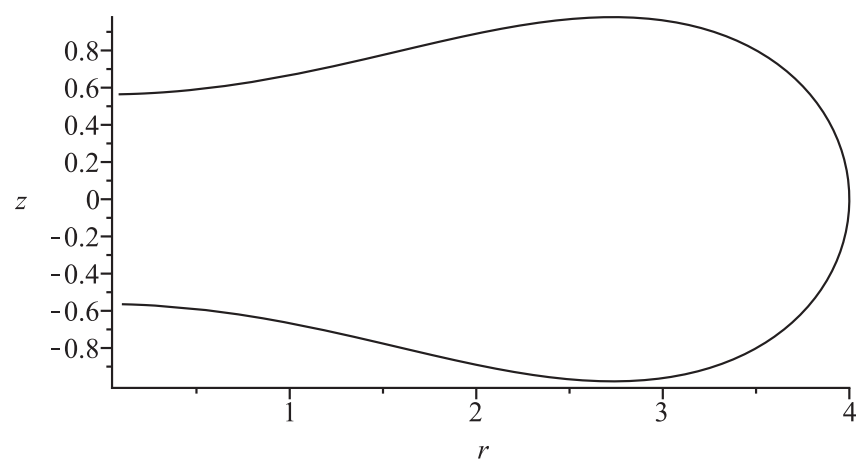

Fig. 3. The profile of the drop generated via (25) with $\stackrel{\circ}{r}=4$ and $a=1 / 0.5333$.

The result of the integration of (7) is

$$
z(r)=\frac{\stackrel{\circ}{2}^{2}-\tilde{m}+\tilde{n}}{2 \sqrt{\tilde{m}}} F(\varphi, k)+\sqrt{\tilde{m}}\left(E(\varphi, k)-\frac{\sin \varphi \sqrt{1-k^{2} \sin ^{2} \varphi}}{1+\cos \varphi}\right),
$$

where

$$
\tilde{m}=\sqrt{\tau^{2}+2 \tau} \stackrel{\circ}{r}^{2}, \quad \tilde{n}=(\tau-1) \stackrel{\circ}{r}, \quad k=\frac{1}{2} \sqrt{\frac{1+2 \tau+2 \sqrt{\tau(\tau+2)}}{\sqrt{\tau(\tau+2)}}},
$$

and

$$
\varphi=\arccos \frac{\tilde{m}-\stackrel{\circ}{r}^{2}+r^{2}}{\tilde{m}+\stackrel{\circ}{r}^{2}-r^{2}} .
$$

We give typical profiles of the rotating drop in Figs. 2 and 3. Note that, throughout this paper (and on the aforementioned Maple worksheet), we have translated the profile curves up to make them symmetric about the horizontal axis.

\section{Parameterization via Weierstrass's functions}

The parametrization presented in the previous section is, however, not quite suitable for a main goal of this paper (i.e., finding closed geodesics on the surface) since it can be used only for visualizing its upper part. For finding geodesics, we need another parametrization which describes the entire surface and that is exactly what we will do below.

Let us start by introducing new parameters by replacing $\tilde{a}$ and $\tilde{c}$ with $a$ and $c$ given by the formulas:

$$
\tilde{a}=\frac{a^{3}}{r}, \quad \tilde{c}=\frac{c}{r}, \quad a \in \mathbb{R}^{+} .
$$

This choice is made because then we have a very simple relation between the new parameters: namely,

$$
c=1-a .
$$


It also turns out to be convenient to introduce the notation

$$
\hat{r}=\frac{r}{r}, \quad \hat{z}=\frac{z}{r}
$$

which allows us to rewrite (7) as

$$
z=\stackrel{\circ}{r} \hat{z}=-\stackrel{\circ}{r} \int \frac{\left(a \hat{r}^{2}+1-a\right) \hat{r} d \hat{r}}{\sqrt{1-\hat{r}^{2}\left(a \hat{r}^{2}+1-a\right)^{2}}} .
$$

This suggests as well that we should change variables in the integral by

$$
\chi=\hat{r}^{2}
$$

transforming the integral into the form:

$$
\hat{z}=-\frac{1}{2} \int \frac{(a(\chi-1)+1) d \chi}{\sqrt{1-\chi(a(\chi-1)+1)^{2}}} .
$$

The expression in the radical is a polynomial of third degree which could be easily converted to the Weierstrass form by replacing the variable $\chi$ with $\xi$ via the formula:

$$
\chi=m \xi+n,
$$

where

$$
m=-\left(\frac{2}{a}\right)^{2 / 3} \quad \text { and } \quad n=\frac{2(a-1)}{3 a} .
$$

In this manner we arrive at an integrand of type

$$
\frac{\mathrm{d} \xi}{\sqrt{4 \xi^{3}-g_{2} \xi-g_{3}}}=\mathrm{d} u
$$

where

$$
g_{2}=\frac{(a-1)^{2}}{3}\left(\frac{2}{a}\right)^{2 / 3}, \quad g_{3}=\frac{(a+2)\left(2 a^{2}-10 a-1\right)}{27 a}
$$

and $u$ is some auxiliary (actually uniformizing) variable.

The integration of (22) is equivalent to inverting the Weierstrass integral of the first kind provided by the Weierstrass $\wp$ function, i.e.,

$$
\xi=\wp\left(u, g_{2}, g_{3}\right)
$$

By making further use of algebra and the properties of the Weierstrass functions, we end up with the expressions

$$
\begin{aligned}
& r(u)=\stackrel{\circ}{r} \sqrt{m \wp\left(u, g_{2}, g_{3}\right)+n} \\
& z(u)=\stackrel{\circ}{r}\left(\frac{a-1}{6}\left(\frac{2}{a}\right)^{2 / 3} u-\left(\frac{2}{a}\right)^{1 / 3} \zeta\left(u, g_{2}, g_{3}\right)\right) .
\end{aligned}
$$

\section{Geodesics on the drop}

Finding closed geodesics on surfaces has a long history in geometry because the existence or non-existence of closed geodesics is intimately tied up with, for instance, the symmetry properties of the surface. In physics, closed geodesics (in the Jacobi metric, see [10]) are trajectories of freely moving particles subject to only the constraint forces keeping them on the surface. Thus, closed geodesics often help us to understand geometrical and physical qualities of a surface which may be the configuration space of a system.

The full parametrization of the rotating drop given in the previous section allows us to visualize geodesics on the rotating drop. Similar visualizations and analyses have appeared in $[5,6,1,2,8,10]$ previously. The key point is 

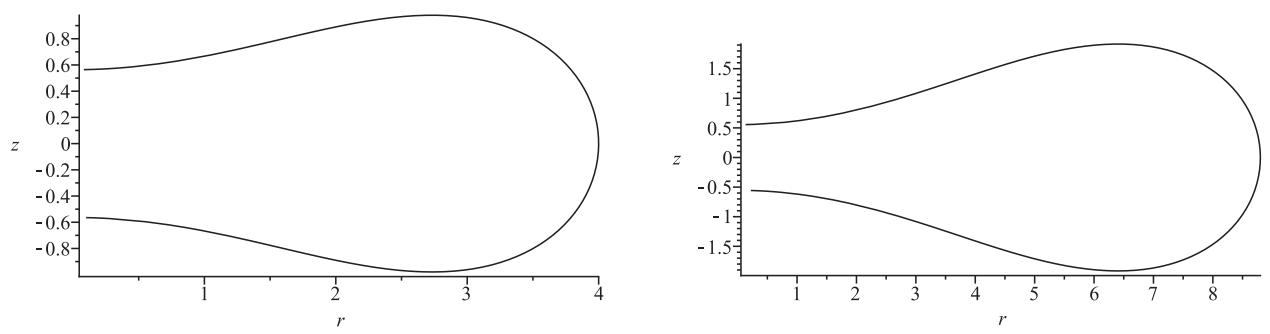

Fig. 4. Profiles of the drop generated via (25) with $\stackrel{\circ}{r}=4, a=1 / 0.53333$ (left) and $\stackrel{\circ}{r}=8.8, a=1 / 0.47$ (right).
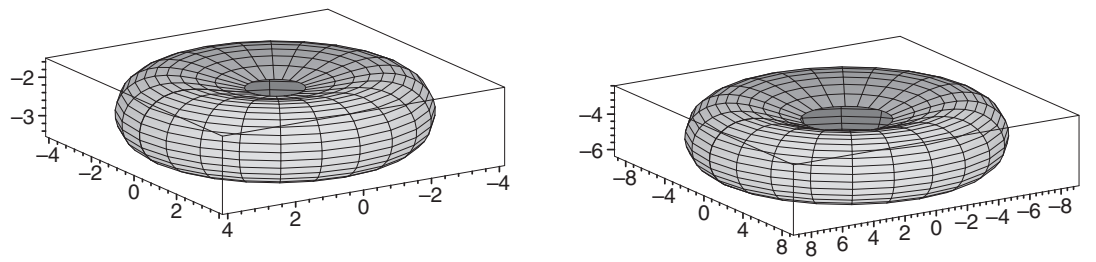

Fig. 5. Rotating drops generated via (25) and parameters $\stackrel{\circ}{r}=4, a=1 / 0.53333$ (left) and $\stackrel{\circ}{r}=8.8, a=1 / 0.47$ (right).

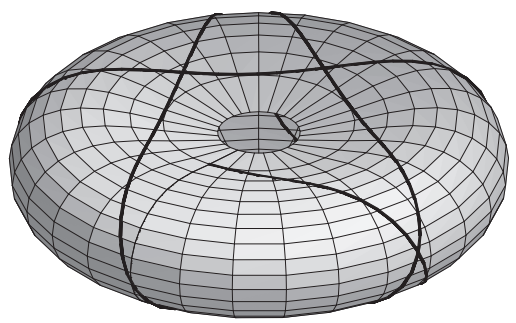

Fig. 6. A wild geodesic on the drop given by (25) with $\stackrel{\circ}{r}=4$ and $a=1 / 0.5333$.

that the Clairaut relation for a surface of revolution confines geodesics starting parallel to parallels to always lie between parallels! This allows for an algorithm to be developed that finds closed geodesics. This algorithm is called "halfbouncepoint"" and it relies on symmetry and the Intermediate Value Theorem to find an initial condition for a closed geodesic. The geodesic is started parallel to a parallel at some $u_{0}$ and then travels halfway around the surface where $u_{1}$ is measured. By symmetry, if the geodesic is closed, then we must have $u_{1}=u_{0}$. The computer algorithm (implemented on Maple) numerically solves for $u_{1}$ and then computes $u_{0}-u_{1}$ for values of $u$ surrounding $u_{0}$. If both positives and negatives occur, then the Intermediate Value Theorem says that there is a point where $u_{1}=u_{0}$ and we have found a closed geodesic. The analyses in [1,2] validate the use of the Intermediate Value Theorem for surfaces of revolution. Maple also provides the means to plot geodesics and the combination of this with "halfbouncepoint" gives an approach to finding closed geodesics on the drop.

First, with the parametrization of the preceding section, we can visualize profile curves and drop surfaces using Maple's Weierstrass functions. See Figs. 4 and 5.

A geodesic plot shows that starting inside the drop hump produces wild geodesics. See Fig. 6.

Here is how we use the procedure "halfbouncepoint". After some preliminary guesses (and plots), we guess that a closed geodesic might be near $u_{0}=2.3$. We now use "halfbouncepoint" to calculate $u_{0}-u_{1}$ as described earlier. We command Maple to carry out the loop

for $\mathrm{jj}$ from -2 to 2 do

$\operatorname{print}(2.292+\mathrm{jj} * 0.0005$, halfbouncepoint $(2.292+\mathrm{jj} * 0.0005,1 / .53333,4))$;

\footnotetext{
2 The procedure halfbouncepoint can also be found on the Maple worksheet at academic.csuohio.edu/oprea_-j/research/research.html or in [10] applied to unduloids.
} 

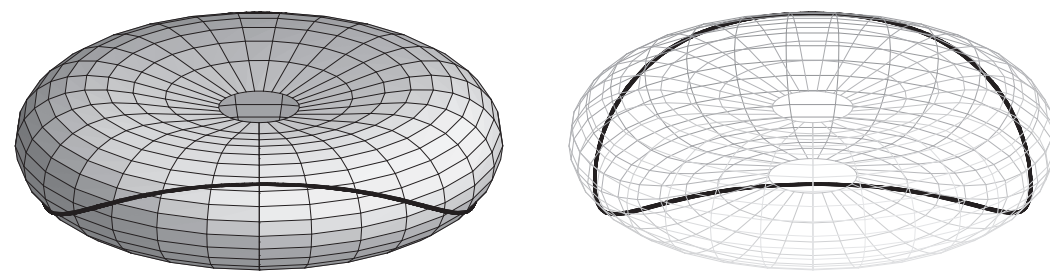

Fig. 7. A closed geodesic on a drop generated via (25) with $\stackrel{\circ}{r}=4$ and $a=1 / 0.5333$.
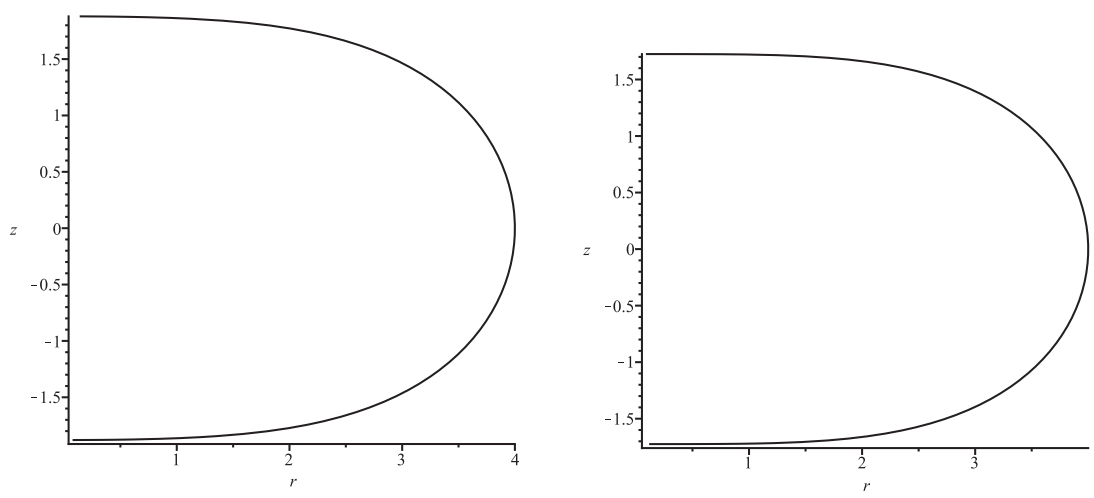

Fig. 8. Profile curves with $\stackrel{\circ}{r}=4: a=0.9$ (left) and $a=1.4$ (right).

The output is as follows:

$$
\begin{gathered}
2.2910,-0.000006415164729 \\
2.2915,-0.000003779765795 \\
2.292,-0.000001700037092 \\
2.2925,-0.0000001733781607 \\
2.2930,0.0000008028376328
\end{gathered}
$$

But now the Intermediate Value Theorem says that we should look around $u_{0}=2.2926$. We zoom in.

for $\mathrm{jj}$ from -2 to 2 do

$\operatorname{print}(2.29265+\mathrm{jj} * 0.00005$, halfbouncepoint $(2.29265+\mathrm{jj} * 0.00005,1 / .53333,4))$;

$$
\begin{gathered}
2.29255,-0.00000005103336997 \\
2.29260,0.00000006581346534 \\
2.29265,0.0000001771657181 \\
2.29270,0.0000002830126378 \\
2.29275,0.0000003833702289
\end{gathered}
$$

So now we try near 2.2926, say 2.29257 and we see that we have an approximation to a closed geodesic. See Fig. 7.

\section{Questions for future work}

The results above leave some questions unanswered. For instance, we seem to have a fundamental difference in shape for surfaces (or, indeed, their profile curves) when the parameter $a$ obeys $a<1$ and $a \geq 1$. For $a<1$, the surfaces are prolate (i.e., the profiles do not have maxima, see Figs. 8 and 9). Yet, from the formulas it is difficult to see why this is true. Even the formulas for Gauss and mean curvatures are simple only for the original parametrization and not for the parametrization using Weierstrass functions. 

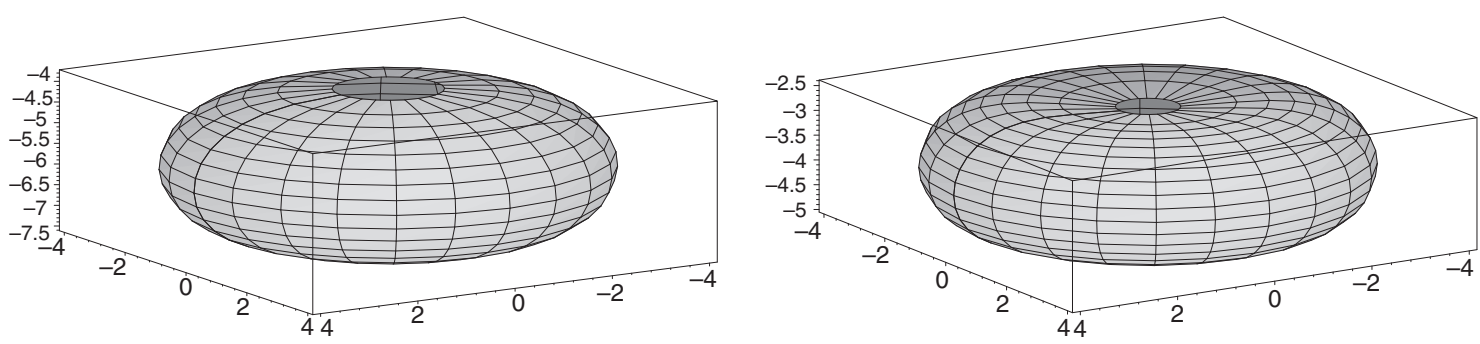

Fig. 9. Drop surfaces with $\stackrel{\circ}{r}=4: a=0.9$ (left) and $a=1.4$ (right).
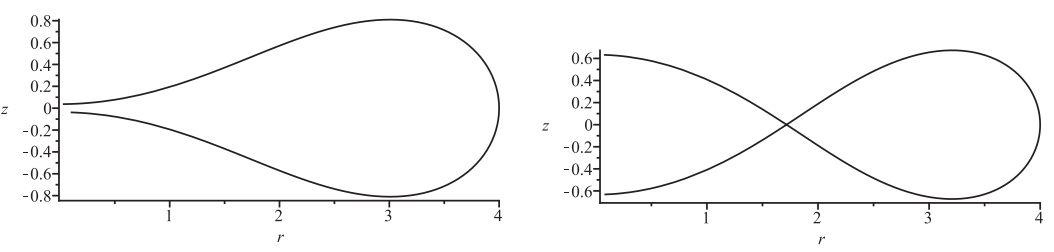

Fig. 10. Profile curves with $\stackrel{\circ}{r}=4: a=2.3$ (left) and $a=2.8$ (right).

Here we show the pictures for $a=1.4$ because they are easier to see than values of $a$ closer to 1 . These elicit the following.

Question 1. What is the explanation for the bifurcation at $a=1$ (sphere) between drop surface shapes? More specifically, why do profile curves for $a<1$ lack maxima (except at $r=0$ of course)?

In principle, we should be able to take $a \in(0,4)$, but we get self-intersecting profile curves when $a$ reaches the critical value $a=2.32911$. This value was found using the FindRoot routine of Mathematica. For this value of $a$, the surface becomes internally tangent to itself and for larger values we get self-intersecting profile curves and their corresponding surfaces (Fig. 10).

Question 2. What is the explanation for the bifurcation around $a=2.32911$ between profile shapes? Furthermore, what is the exact bifurcation parameter $a$ from a theoretical (i.e., analytical rather than numerical) viewpoint?

Finally, in regard to geodesics, we see another difference in behavior between the cases $a \leq 1$ and $a>1$. As we have seen, for $a>1$, we can show the existence of non-equatorial closed geodesics and then "find" them using numerical solutions of the geodesic equations and fundamental theorems such as the Intermediate Value Theorem. For $a<1$, we have been unsuccessful in finding any closed geodesic other than the equator. Of course, our approach to finding closed geodesics only looks for ones that start parallel to parallels, so perhaps there are others (Fig. 11).

Question 3. Are there closed non-equatorial geodesics on drop surfaces with $a<1$ ?

Perhaps the methods of [1] may say something here by analyzing the Weierstrass functions in this context. The Clairaut relation in this context says that, if we start at $u=\bar{u}$ and parallel to a parallel, then along the geodesic it must always be true that $G(u) \geq G(\bar{u})$, where $G(u)$ is the metric coefficient $G$ at parameter value $u$. (Recall that a surface of revolution with revolution parameter $v$ and profile curve parameter $u$ has the property that the metric coefficients $E$

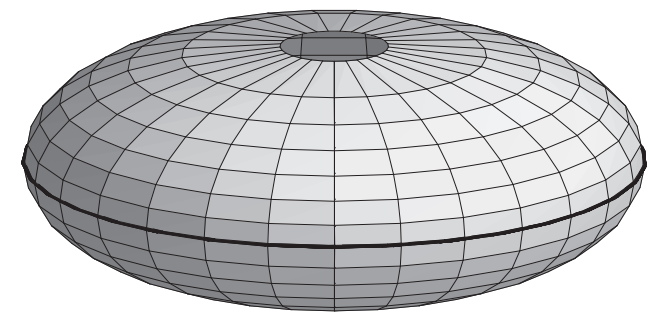

Fig. 11. The equatorial geodesic on a drop with $\stackrel{\circ}{r}=4$ and $a=2 / 3$. 
and $G$ only depend on $u$ and the metric coefficient $F=0$.) The $G$ here (which is obtained from a bit of simplification applied to the result of the calculations introduced earlier) is

$$
G=-\left(\left(\frac{2}{a}\right)^{2 / 3} \cdot \wp\left(u, g_{2}, g_{3}\right)-\frac{2 a-2}{3 a}\right) \stackrel{\circ}{r}^{2}
$$

which can be easily recognized as $r^{2}(u)$ from (25) and which is in complete agreement with the expression given in (8). This means that a geodesic starting parallel to a parallel will always be confined to the region with $r(u) \geq r(\bar{u})$; that is, the geodesic stays at least as far from the axis of revolution as the starting point is.

The more surprising result produced by Maple is that $E$ takes the form

$$
E=\frac{1}{\left(2 a^{2}\right)^{2 / 3}} \frac{\stackrel{\circ}{r}}{r^{2}(u)} .
$$

But now we have the result.

Theorem. The surface area $\mathcal{A}$ of the rotating drop with parameters a and $\stackrel{\circ}{r}$ is

$$
\mathcal{A}=2 \pi\left(\frac{2}{a}\right)^{2 / 3} \stackrel{\circ}{u} \stackrel{\circ}{r}^{2}
$$

where $\stackrel{\circ}{u}$ satisfies $r(\stackrel{\circ}{u})=\stackrel{\circ}{r}$.

Proof. Since the metric coefficient $F$ vanishes for a surface of revolution, the surface area is given by

$$
2 \int_{0}^{2 \pi} \int_{0}^{\stackrel{\circ}{u}} \sqrt{E G} \mathrm{~d} u \mathrm{~d} v
$$

where the factor 2 accounts the symmetric bottom half of the drop. But by the above, we see that $\sqrt{E G}=\stackrel{\circ}{2}^{2} /\left(2 a^{2}\right)^{1 / 3}$, a constant. The integration is then trivial.

The fact that the total surface area of the drop is proportional to $\stackrel{\circ}{u}$ is quite unusual and interesting.

Question 4. Does the formula for surface area have any physical consequences? Find other surfaces with this relationship. Does the proportionality of surface area to $\stackrel{\circ}{u}$ characterize a class of surfaces?

We have seen that physical systems produce interesting geometry and that this geometry can be studied if we can parametrize properly - even if we must use complicated functions such as Weierstrass elliptic functions. We have also seen that computers and modern computer algebra software can provide insights through visualization and computation that might have eluded us in our youth.

\section{References}

[1] J. Alexander, Closed geodesics on certain surfaces of revolution, J. Geom. Symmetry Phys. 8 (2006) 1-16.

[2] J. Borzellino, C. Jordan-Squire, G. Petrics, D. Sullivan, Closed geodesics on orbifolds of revolution, Houston J. Math. 33 (2007) $1011-1025$.

[3] W. Kühnel, Differential Geometry, Curves - Surfaces - Manifolds, American Mathematical Society, Providence, R.I., 2002.

[4] W. Kühnel, M. Steller, On closed Weingarten surfaces, Monatsh. Math. 146 (2005) 113-126.

[5] I. Mladenov, J. Oprea, The mylar balloon revisited, Am. Math. Monthly 110 (2003) 761-784.

[6] I. Mladenov, J. Oprea, Unduloids and their closed geodesics, in: Proceedings of the Fourth International Conference on Geometry, Integrability and Quantization, Coral Press, Sofia, 2003, pp. 206-234.

[7] I. Mladenov, J. Oprea, On some deformations of the mylar balloon, in: Proceedings of the XV International Workshop on Geometry and Physics, vol. XX, Publ. De La RSME, Puerto De La Cruz, Tenerife, Canary Islands, 2007, pp. 310-315.

[8] A. Mourad, I. Moukadem, Characterization and computation of closed geodesics on toroidal surfaces, J. Geom. Symmetry Phys. 16 (2009) 23-37.

[9] J. Oprea, The Mathematics of Soap Films: Explorations with Maple, American Mathematical Society, Providence, R.I., 2000.

[10] J. Oprea, Differential Geometry and Its Applications, Mathematical Association of America, Washington, DC, 2007. 